\title{
Interventions for erythema nodosum leprosum (Protocol)
}

\author{
Lockwood D, van Brakel WH, Ramirez Jr J, Richardus JH, Van Veen NHJ
}

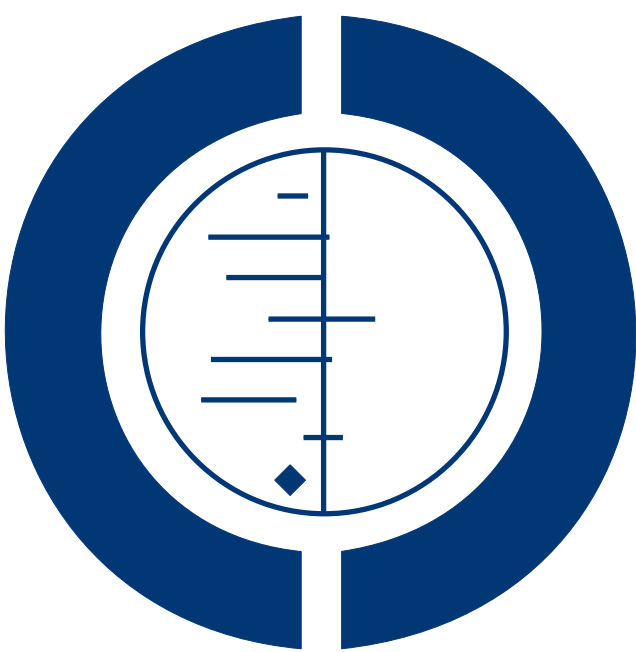

THE COCHRANE COLLABORATION $^{\circledR}$

This is a reprint of a Cochrane protocol, prepared and maintained by The Cochrane Collaboration and published in The Cochrane Library 2008, Issue 1

http://www.thecochranelibrary.com

\section{WILEY}


TABLE OF CONTENTS

HEADER . . . . . . . . . . . . . . . . . . . . . . . . . . . . . . . . . . . . 1

ABSTRACT . . . . . . . . . . . . . . . . . . . . . . . . . . . . . . . . . . . . . . . . . . . . .

BACKGROUND . . . . . . . . . . . . . . . . . . . . . . . . . . . . . . . . . . . .

OBJECTIVES . . . . . . . . . . . . . . . . . . . . . . . . . . . . . . . . . . . . . . . . . . .

METHODS . . . . . . . . . . . . . . . . . . . . . . . . . . . . . . . . . . . . . . . .

ACKNOWLEDGEMENTS . . . . . . . . . . . . . . . . . . . . . . . . . . . . . . . . . . . . . . . .

REFERENCES . . . . . . . . . . . . . . . . . . . . . . . . . . . . . . . . . . . . . . 6

WHAT'S NEW . . . . . . . . . . . . . . . . . . . . . . . . . . . . . . . . . . . . . 7

HISTORY . . . . . . . . . . . . . . . . . . . . . . . . . . . . . . . . . . . . . . . 7

CONTRIBUTIONS OF AUTHORS . . . . . . . . . . . . . . . . . . . . . . . . . . . . . . . . . . . . . . . . . . .

DECLARATIONS OF INTEREST . . . . . . . . . . . . . . . . . . . . . . . . . . . . . . . . . . . . . . .

SOURCES OF SUPPORT . . . . . . . . . . . . . . . . . . . . . . . . . . . . . . . . . . . . . . . . . . . .

Interventions for erythema nodosum leprosum (Protocol)

Copyright $\odot 2009$ The Cochrane Collaboration. Published by John Wiley \& Sons, Ltd. 


\title{
[Intervention Protocol]
}

\section{Interventions for erythema nodosum leprosum}

\author{
Diana Lockwood ${ }^{2}$, Wim H van Brakel ${ }^{3}$, Jose Ramirez Jr ${ }^{4}$, Jan Hendrik Richardus ${ }^{1}$, Natasja HJ Van Veen ${ }^{1}$ \\ ${ }^{1}$ Department of Public Health, Erasmus MC, University Medical Center Rotterdam, Rotterdam, Netherlands. ${ }^{2}$ Clinical Research \\ Unit \& Tropical Medicine , London School of Hygiene \& Tropical Medicine, London, UK. ${ }^{3}$ Leprosy Unit, Royal Tropical Institute, \\ Amsterdam, Netherlands. ${ }^{4} \mathrm{c} / \mathrm{o}$ Cochrane Skin Group, University of Nottingham, Nottingham, UK
}

Contact address: Natasja HJ Van Veen, Department of Public Health, Erasmus MC, University Medical Center Rotterdam, PO Box 2040, Rotterdam, 3000 CA, Netherlands.n.vanveen@erasmusmc.nl.

Editorial group: Cochrane Skin Group.

Publication status and date: Unchanged, published in Issue 2, 2009.

Citation: Lockwood D, van Brakel WH, Ramirez Jr J, Richardus JH, Van Veen NHJ. Interventions for erythema nodosum leprosum. Cochrane Database of Systematic Reviews 2008, Issue 1. Art. No.: CD006949. DOI: 10.1002/14651858.CD006949.

Copyright (C) 2009 The Cochrane Collaboration. Published by John Wiley \& Sons, Ltd.

\begin{abstract}
A B S T R A C T
This is the protocol for a review and there is no abstract. The objectives are as follows:

To assess the effects of any therapy or treatment used in the management of ENL.
\end{abstract}

\section{B A C K G ROU N D}

\section{Description of the condition}

Leprosy is a chronic infectious disease caused by the bacillus $M y$ cobacterium (M.) leprae. Leprosy bacilli are spread as tiny droplets from the nose or mouth of infected and untreated individuals. When the immune system fails to respond effectively, the disease will develop. Often, the first sign of leprosy is a patch on the skin, but damage to peripheral nerves may occur as well. Leprosy can appear in various clinical forms, dependent on the response of the immune system. Some people have only a few skin patches and the number of bacilli is relatively small. This is classified as paucibacillary $(\mathrm{PB})$ leprosy. Other people have many skin patches and a high number of bacilli in their body and are classified as multibacillary (MB) leprosy (ILEP 2001; WHO 2006a). At the beginning of 2006 the prevalence was about 220,000 worldwide. The number of newly detected cases was approximately 296,000 during 2005 (WHO 2006b).
The body's immune response to the leprosy bacilli may also cause 'so-called' reactions. There are two types of reactions: type 1 reaction or reversal reaction (RR) and type 2 reaction or erythema nodosum leprosum (ENL). Type 1 or RR presents as acute inflammation in skin lesions and nerves. ENL presents as new, red, painful and tender swellings in the skin, usually on the legs and arms, and sometimes on the trunk. ENL varies in severity. When the reaction is mild, only the skin is affected and there may be lowgrade fever. When the reaction is severe, the swellings are multiple and may ulcerate, and other organs may be inflamed, such as the nerves, eyes, joints, testes, and lymph nodes (ILEP 2002; WHO 2003). Most people with ENL have recurrent episodes which may occur over a period of several years. Few people experience a single acute episode of ENL (Nery 1998; Saunderson 2000; Pocaterra 2006).

ENL only occurs in people with MB leprosy, especially in those classified as borderline lepromatous (BL) or lepromatous (LL) leprosy. These people have a high number of bacilli in their body which increases the risk of ENL. The percentage of people diagnosed with ENL seems to differ between countries and studies. 
For instance, a study from Ethiopia reported only 5\% ENL among people with $\mathrm{MB}$ leprosy, while a study from Brasil found an incidence of 31\% (Nery 1998; Saunderson 2000). ENL may occur before the start of treatment, but develops most often within the first three years after the start of multi drug therapy (MDT). After completion of treatment, people may still have episodes of ENL for several years. This depends mainly on the number of bacilli remaining in the body after treatment (ILEP 2002; Naafs 2003a).

\section{Causes}

ENL is caused partly by immune complexes of $M$. leprae antigen and antibody. These complexes circulate in the blood and may precipitate in tissue, particularly on the wall of small blood vessels, causing acute inflammation (vasculitis) and release of tissue-damaging enzymes. In addition, the immune system activates cells (macrophages and $\mathrm{T}$ cells) that attack and kill the bacilli (Lockwood 1996; Naafs 2003b). Studies have shown that some people have a higher risk of getting ENL, such as people with lepromatous leprosy or with many bacilli, and people aged 40 or younger (Manandhar 1999; Saunderson 2000; Kumar 2004).

\section{Impact}

People who have ENL usually feel ill (general malaise, fever) and many organs may be affected. ENL is often a recurrent or chronic condition and requires treatment for a long period (Nery 1998; Saunderson 2000; Pocaterra 2006). More than most other diseases, leprosy has a very negative image. Having visible signs of leprosy or side-effects from treatment can trigger discrimination and stigmatization (Heijnders 2004; Rafferty 2005). The psychological impact of a chronic and stigmatizing condition may be profound.

\section{Description of the intervention}

Most therapies for ENL aim to control the acute inflammation, relieving the pain and preventing further damage or new episodes. Several treatments are available for ENL.

The conventional treatment for mild ENL is rest and anti-inflammatory medication. Aspirin is the most commonly used anti-inflammatory, but indomethacin, chloroquine and colchicine have been tested as well. There is not much evidence that these drugs are more beneficial than aspirin (ILEP 1996; Lockwood 1996).

For severe ENL, prednisolone, the most widely available corticosteroid, and clofazimine are most commonly used. Prednisolone usually acts rapidly in controlling ENL. The starting dose should be the lowest possible to control ENL and gradually reduced as soon as possible. The schedule for reducing prednisolone depends on the course of the disease. ENL is often recurrent or chronic and requires high-dose and prolonged courses of prednisolone for disease control. This increases the risk of adverse events, such as hypertension or diabetes, and steroid dependency (ILEP 1996; Lockwood 1996; ILEP 2002).

Leprosy infection can be effectively treated with a combination of antibiotics. Multidrug therapy (MDT) with the antibiotics rifampicin, dapsone and clofazimine was introduced in the 1980s and is provided free by the World Health Organization (WHO) (ILEP 2001; WHO 2006a). Since the introduction of MDT, the number of people affected by leprosy has decreased substantially. Before the introduction of MDT, as many as half of those with MB leprosy developed ENL (Lockwood 1996; Saunderson 2000). Since MDT has been provided the number of new people with ENL has decreased substantially. This reduction is probably due to earlier diagnosis and treatment and to the effect of clofazimine, one of the drugs used in MDT for MB leprosy (Pocaterra 2006). Clofazimine is a useful drug when corticosteroids are contraindicated or need to be reduced. Treatment with clofazimine usually needs more time and higher doses, because it is less effective than corticosteroids. The dose of clofazimine needed to control ENL is higher than the dose used in MDT. Disadvantages of continuous high doses of clofazimine are gastrointestinal symptoms (e.g. diarrhoea) and brown discoloration of the skin (ILEP 1996; Lockwood 1996; WHO 2003).

Another highly effective, but controversial, drug in the management of ENL is thalidomide. The major problem with thalidomide is that it may cause serious birth defects when taken in early pregnancy. Therefore, the WHO does not support the use of this drug (ILEP 1996; WHO 2003).

Other therapies have been tested, such as cyclosporin A, pentoxifylline, oral zinc, and Mycobacterium $w$ vaccination (Uyemura 1986; Zaheer 1993; Mahajan 1994; Nery 2000). New therapies for other immune-mediated conditions seem promising. Examples are TNF- $\alpha$ antibody treatment, intravenous immunoglobulin, and tenidap (Lockwood 1996). It is plausible that these therapies may be effective for controlling ENL, but evidence from randomized controlled trials is very limited.

\section{Why it is important to do this review}

ENL is a serious immunological complication of leprosy. The complex mechanisms underlying ENL are not fully understood yet which makes treatment difficult. Corticosteroids and clofazimine are the drugs of choice for ENL, but both have drawbacks and the optimal regimen has not been established. Alternative therapies have been tested, but it is unclear if they are beneficial, or which one is preferable. The role of newer treatments, such as TNF- $\alpha$ antibody treatment, intravenous immunoglobulin, and tenidap, is not known.

\section{O B J E C T I VES}


To assess the effects of any therapy or treatment used in the management of ENL.

\section{METHODS}

\section{Criteria for considering studies for this review}

\section{Types of studies}

All randomised controlled trials (RCTs) of any design.

\section{Types of participants}

Anyone affected by leprosy with ENL, confirmed by appropriate clinical features. The definition of ENL is: 'an inflammatory condition involving the humoral immune system, in which people develop crops of tender erythematous subcutaneous skin lesions'. There may be accompanying neuritis, iritis (inflammation of the iris), arthritis, orchitis (inflammation of the testicles), dactylitis (inflammation of the fingers and toes), lymphadenopathy, oedema and fever. The skin signs are obligatory; the nerve and general signs optional (Smith 2002; Van Brakel 2005).

\section{Types of interventions}

Any therapy for ENL, including:

\section{(1) Systemic corticosteroids}

- Oral therapies: prednisolone

- Intravenous therapies: betamethasone, methylprednisolone

\section{(2) Systemic non-steroidal immunomodulatory therapies}

- Immunosuppressive therapies: thalidomide, cyclosporin A, pentoxifylline

- Antibacterial therapies: clofazimine

- Anti-inflammatory therapies: aspirin, chloroquine, colchicine, indomethacin

\section{(3) Diverse therapies}

- Oral zinc

- Mycobacterium $w$ vaccine

The comparators will be no treatment, placebo, usual care (e.g. systemic corticosteroid with or without pentoxifylline) or another listed therapy. We shall include trials which compare different dosages of the same therapy or different routes of administration (e.g. intravenous versus oral systemic corticosteroids).

\section{Types of outcome measures}

Primary outcomes

(i) The proportion of participants achieving remission of skin lesions

Remission is defined as the absence of new tender erythematous subcutaneous skin lesions at completion of the ENL therapy, as assessed by a clinician.

\section{Secondary outcomes}

(ii) The proportion of participants achieving remission of other inflammations

Remission is defined as the disappearance of other inflammations associated with ENL (e.g. iritis, arthritis) at completion of the ENL therapy, as assessed by a clinician.

\section{(iii) Investigator-assessed change in ENL severity}

The change in ENL severity, compared to baseline, using a grading scale as used in each of the studies.

\section{(iv) Time to next clinical episode of ENL}

Time to next clinical episode of ENL is defined as the time between the last dose of ENL treatment and appearance of new signs of ENL reaction.

\section{(v) Changes in quality of life}

As assessed using a recognised instrument (generic, dermatology specific, disease specific, or patient-generated index).

\section{Adverse outcomes}

We are looking at a very wide range of interventions and cannot pre-specify which are the most important / common adverse events. Therefore we shall document the incidence and severity of all recorded local and systemic adverse events, at any time point, in all the included studies.

\section{Economic data}

Data relating to costs will not be reported, but we will address cost implications in the discussion. 


\section{Timing of outcome assessment}

Data that has been recorded for less than four weeks from the start of treatment will be considered to reflect short-term benefit and will be analysed separately from data that was recorded for longer than three months from the start of treatment, which we consider to reflect the minimum time period to capture any longer-term benefit. The short-term assessment (one to four weeks) will be considered the primary endpoint, because the definite treatment effects should be visible within the first weeks. The long-term assessment (three to six months) will be used as a secondary endpoint.

\section{Search methods for identification of studies}

\section{Electronic searches}

We shall search for relevant published trials in:

- the Cochrane Skin Group Specialised Register, and the Cochrane Central Register of Controlled Trials in The Cochrane Library (last update);

- MEDLINE (from 2003) and EMBASE (from 2005),

- AMED (Allied and Complementary Medicine, from 1985), CINAHL (from 1980), and LILACS (Latin American and Caribbean Health Science Information database, from 1982).

We shall search for ongoing trials in:

- the metaRegister of Controlled Trials (www.controlledtrials.com);

- the ongoing trials website of the Cochrane Skin Group (www.nottingham.ac.uk/csg/about/ongoingtrial).

\section{(i) Search strategy to locate RCTs}

Search terms 1-29, as given in the Cochrane Handbook for Systematic Reviews of Interventions (Higgins 2005), Appendix 5b.2.

\section{(ii) Strategy to locate erythema nodosum leprosum (ENL)}

\section{0. leprosy.mp. or exp LEPROSY/}

31. type 2 reaction.mp.

32. lepra reaction.mp.

33. ENL.mp.

34. *Erythema Nodosum/

35. LEPROSY, BORDERLINE/

36. LEPROSY, LEPROMATOUS/

37.30 and 31

38.30 and 33

39.32 or 34 or 35 or 36 or 37 or 38

40. 29 and 39

The results of searches (i) and (ii) will be combined with the Boolean operator 'AND'.
This is a draft search strategy and will be adapted to include additional search terms where necessary and will be modified for the other databases listed.

\section{Searching other resources}

\section{Reference lists}

We shall scan the bibliographies of the included studies and reviews for possible references to RCTs.

\section{Unpublished literature}

We shall attempt to find unpublished or ongoing trials via correspondence with trial authors of included and excluded trials less than 15 years old.

\section{Handsearching}

Conference proceedings from relevant leprosy meetings will be scanned for RCTs and, where possible, the authors will be contacted for further information.

\section{Adverse effects}

We shall not do a separate search for adverse events, however, we shall search within the included studies.

\section{Language restrictions}

No language restrictions will be imposed when searching for publications, and translations will be sought where necessary.

\section{Data collection and analysis}

\section{Selection of studies}

Two authors (NvV and JHR) will check the titles and abstracts identified from the searches. If it is clear that the study does not refer to a randomised controlled trial of an ENL intervention, we shall exclude it. The same two authors will independently assess the full text version of each remaining study to determine whether it meets the pre-defined selection criteria. Any differences of opinion will be resolved through discussion within the review team. We shall list the excluded studies and reasons for exclusion in the 'Characteristics of excluded studies' table. These will not be discussed further.

Interventions for erythema nodosum leprosum (Protocol)

Copyright $\odot 2009$ The Cochrane Collaboration. Published by John Wiley \& Sons, Ltd. 


\section{Data extraction and management}

Two authors (NvV and JHR) will independently extract the data using a specially designed data extraction form. A third team member (WvB) will resolve any differences in opinion. Two authors ( $\mathrm{NvV}$ and JHR) will independently check and enter the data into RevMan.

For the participant's and investigator's global assessments of improvement, the authors will translate reported changes in ENL severity into the proportion of participants with improvement greater than minimal. By minimal we mean anything greater than the first category of improvement on a Likert scale, or greater than $50 \%$ improvement from baseline on a continuous scale. For the purpose of calculating clinical efficacy, we shall regard categories relating to greater than minimal improvement as a treatment success.

All other outcomes will be expressed as the actual or percentage change from baseline.

\section{Assessment of risk of bias in included studies}

The quality assessment will include an evaluation of the following components for each included study, since there is some evidence that these are associated with biased estimates of treatment effect (Juni 2001):

(a) the method of generation of the randomisation sequence;

(b) the method of allocation concealment - it will be considered 'adequate' if the assignment could not be foreseen;

(c) who was blinded and not blinded (participants, clinicians, outcome assessors) if this is appropriate;

(d) how many participants were lost to follow up in each arm, and whether reasons for losses were adequately reported;

(e) whether all participants were analysed in the groups to which they were originally randomised (intention to treat principle).

In addition we shall report on:

(f) the degree of certainty that the participants have ENL;

(g) the baseline assessment of the participants for age, sex, duration and severity of ENL;

(h) whether outcome measures were described.

We shall describe the quality of each study, based on these components, in the section on Methodological quality of included studies. We shall also record this information in a 'Table of quality criteria'.

\section{Measures of treatment effect}

We shall express the results as risk ratio (RR) and 95\% confidence intervals (CI) for dichotomous outcomes, and differences in means (MD) and 95\% CI for continuous outcomes. We shall use a standardised mean difference to combine continuous outcome measures, since they are likely to be from different scales across the studies, such as quality of life. We shall express the result as 'number needed to treat' where appropriate, for a range of plausible control event rates. For time to event outcomes, the log hazard ratio and $95 \%$ confidence interval will be summarised.

\section{Unit of analysis issues}

Where there are multiple intervention groups within a trial, we shall make pairwise comparisons of similar ENL interventions or ENL active components versus no treatment, placebo, or another ENL intervention. We shall analyse cross-over trials using data from the first phase only and pooled, where possible, with parallel design studies. We shall analyse internally controlled trials using appropriate techniques for paired designs and these studies will not be pooled with studies of other designs. We shall exclude nonrandomised controlled studies from the analyses but these may be commented on in the discussion.

\section{Dealing with missing data}

If participant drop out leads to missing data, we shall conduct an intention-to-treat analysis. We shall contact trial authors or sponsors of studies less than 15 years old to provide missing statistics such as standard deviations. For dichotomous outcomes, we shall regard participants with missing outcome data as treatment failures and include these in the analysis. For continuous outcomes, we shall carry forward the last recorded value for participants with missing outcome data.

\section{Data synthesis}

For studies with a similar type of ENL intervention or a similar active component, we shall perform a meta-analysis to calculate a weighted treatment effect across trials, using a random-effects model. Where it is not possible to perform a meta-analysis we shall summarise the data for each trial.

\section{Subgroup analysis and investigation of heterogeneity}

We shall assess statistical heterogeneity using $\mathrm{I}^{2}$. If substantial heterogeneity $\left(\mathrm{I}^{2}>50 \%\right)$ exists between studies for the primary outcome, we shall explore the reasons for heterogeneity; such as disease severity, dosage and duration of treatment. We shall perform further subgroup analysis where adequate information is given. The groups will be different severity of ENL (mild or severe), and different duration of ENL (single acute, multiple acute or chronic).

\section{Sensitivity analysis}

We plan to conduct sensitivity analyses to examine the effects of excluding poor quality studies, defined as those with a moderate 
or high risk of bias as described in the Cochrane Handbook of Systematic Reviews of Interventions (Higgins 2005).
Where there is uncertainty, we shall contact the trial authors for clarification. A consumer is part of the review team to ensure the relevance and readability of the final review.

\section{Adverse outcomes}

We shall describe the information qualitatively.

\section{ACKNOWLEDGEMENTS}

Other

The editorial base would like to thank Alireza Firooz (external expert) and Jack Tweed (consumer).

\section{REFEREN CES}

\section{Additional references}

\section{Heijnders 2004}

Heijnders ML. The dynamics of stigma in leprosy. International Journal of Leprosy and Other Mycobacterial Diseases 2004;72(4):437-47.

\section{Higgins 2005}

Higgins JPT, Green S, editors. Cochrane Handbook for Systematic Interventions 4.2.5 [updated May 2005]. The Cochrane Library, Issue 3. Chichester, UK: John Wiley \& Sons, Ltd, 2005.

\section{ILEP 1996}

The International Federation of Anti-Leprosy Associations (ILEP). The management of erythema nodosum leprosum. ILEP Technical Bulletin 1996, issue 9.

\section{ILEP 2001}

The International Federation of Anti-Leprosy Associations (ILEP). How to diagnose and treat leprosy. London: ILEP, 2001.

\section{ILEP 2002}

The International Federation of Anti-Leprosy Associations (ILEP). How to recognise and manage reactions. London: ILEP, 2002.

\section{Juni 2001}

Juni P, Altman DG, Egger M. Systematic reviews in health care: assessing the quality of controlled clinical trials. BMJ 2001;323:42-6.

\section{Kumar 2004}

Kumar B, Dogra S, Kaur I. Epidemiological characteristics of leprosy reactions: 15 years experience from north India. International Journal of Leprosy and Other Mycobacterial Diseases 2004;72(2):125-33.

\section{Lockwood 1996}

Lockwood DN. The management of erythema nodosum leprosum: current and future options. Leprosy Review 1996; 67(4):253-9.

Mahajan 1994

Mahajan PM, Jadhav VH, Patki AH, Jogaikar DG, Mehta JM. Oral zinc therapy in recurrent erythema nodosum leprosum: a clinical study. Indian Journal of Leprosy 1994; 66(1):51-7.

\section{Manandhar 1999}

Manandhar R, LeMaster JW, Roche PW. Risk factors for erythema nodosum leprosum. International Journal of Leprosy and Other Mycobacterial Diseases 1999;67(3):270-8.

Naafs 2003a

Naafs B. Reactions: the body as battlefield I: clinical aspects. Memisa Medisch 2003;69(2):330-6.

\section{Naafs 2003b}

Naafs B. Reactions: the body as battlefield II: immunopathology. Memisa Medisch 2003;69(2):337-42.

\section{Nery 1998}

Nery JA, Vieira LM, de Matos HJ, Gallo ME, Sarno EN. Reactional states in multibacillary Hansen disease patients during multidrug therapy. Revista do Instituto de Medicina Tropical de São Paulo 1998;40(6):363-70.

\section{Nery 2000}

Nery JA, Perisse AR, Sales AM, Vieira LM, Souza RV, Sampaio EP, et al.The use of pentoxifylline in the treatment of type 2 reactional episodes in leprosy. Indian Journal of Leprosy 2000;72(4):457-67.

\section{Pocaterra 2006}

Pocaterra L, Jain S, Reddy R, Muzaffarullah S, Torres O, Suneetha $S$, et al.Clinical course of erythema nodosum leprosum: an 11-year cohort study in Hyderabad, India. American Journal of Tropical Medicine and Hygiene 2006;74 (5):868-79.

Rafferty 2005 Rafferty J. Curing the stigma of leprosy. Leprosy Review 2005;76(2):119-26.

\section{Saunderson 2000}

Saunderson P, Gebre S, Byass P. ENL reactions in the multibacillary cases of the AMFES cohort in central Ethiopia: incidence and risk factors. Leprosy Review 2000; 71(3):318-24.

\section{Smith 2002}

Smith WC, Nicholls PG. Special workshop on repeated and late reactions. International Journal of Leprosy and Other Mycobacterial Diseases 2002;70(4):339-41. 
Uyemura 1986

Uyemura K, Dixon JF, Wong L, Rea TH, Modlin RL. Effect of cyclosporine A in erythema nodosum leprosum. Journal of Immunology 1986;137(11):3620-3.

Van Brakel 2005

van Brakel WH, Nicholls PG, Das L, Barkataki P, Suneetha SK, Jadhav RS, et al.The INFIR Cohort Study: investigating prediction, detection and pathogenesis of neuropathy and reactions in leprosy. Methods and baseline results of a cohort of multibacillary leprosy patients in north India. Leprosy Review 2005;76(1):277-95.

WHO 2003

World Health Organization. WHO Guidelines for the management of severe erythema nodosum leprosum (ENL) reactions. World Health Organization 2003.

\section{WHO 2006a}

World Health Organization. Leprosy. http://www.who.int/ mediacentre/factsheets/fs101/en/index.html (Accessed 27 November 2006).

\section{WHO 2006b}

World Health Organization. Global leprosy situation, 2006. Weekly Epidemiological Record 2006;81(32):309-16.

\section{Zaheer 1993}

Zaheer SA, Misra RS, Sharma AK, Beena KR, Kar HK,

Mukherjee A, et al.Immunotherapy with Mycobacterium w vaccine decreases the incidence and severity of type 2 (ENL) reactions. Leprosy Review 1993;64(1):7-14.

* Indicates the major publication for the study

\section{WHAT'S NEW}

Date Event Description

6 June 2008 Amended Converted to new review format.

\section{H I S T O R Y}

Protocol first published: Issue 1, 2008

\section{CONTRIBUTIONSOFAUTHORS}

Link with editorial base and co-ordinate contributions from co-authors $(\mathrm{NvV})$

Draft protocol (NvV, JHR, DL, WVB, JR)

Run search (vVV)

Identify relevant titles and abstracts from searches ( $\mathrm{NvV}, \mathrm{JHR})$

Obtain copies of trials $(\mathrm{NvV}, \mathrm{JHR})$

Selection of trials (NvV, JHR)

Extract data from trials ( $\mathrm{NvV}$, JHR)

Enter data into RevMan ( NvV, JHR)

Carry out analysis ( NvV, JHR, WVB)

Interpret data $(\mathrm{NvV}, \mathrm{JHR}, \mathrm{WvB}, \mathrm{DL})$

Draft final review ( NvV, JHR, DL, WvB, JR)

Update review ( $\mathrm{NvV}$ JHR, DL, WvB, JR) 


\section{DECLARATIONSOF INTEREST}

DNL: has been paid advisor to the drug company Pharmion (who makes Thalidomide) advising them on their application to have Thalidomide registered within the EU.

\section{SOURCES OF SUPPORT}

\section{Internal sources}

- The Netherlands Leprosy Relief, Netherlands.

\section{External sources}

- No sources of support supplied 